\section{Response to Koeller et al.}

To the Editor: In their letter, Koeller et al. ${ }^{1}$ state that our review $^{2}$ of the available evidence on the CPT1A P479L allele reflects "a misunderstanding of causation assessment in epidemiological studies." We believe they have misread our analysis. As they note, observational, nonrandomized epidemiological association studies alone are not sufficient for an inference of causality. When such studies demonstrate a statistically significant relationship, as is the case for the P479L/P479L genotype and infant death, additional evidence must be considered in determining whether the association is causal and whether it warrants public health intervention. To accomplish the analysis of causation, we applied a wellestablished set of criteria from Koepsell and Weiss's Epidemiologic Methods: Studying the Occurrence of Illness. ${ }^{3}$ As discussed in our paper, this analysis not only includes a critical assessment of the observational data for strength and consistency but also examines factors such as biological plausibility and alternative explanations. These latter criteria were important in concluding that existing evidence could not establish the P479L/P479L genotype as a causal factor in infant death.

Two factors related to biological plausibility deserve particular emphasis. The first is that the proposed risk genotype (P479L/P479L) is very common: it occurs in more than $50 \%$ of individuals in most of the northern indigenous populations where the association with infant death has been noted, with no evident difference in prevalence between newborns and adults. Thus, while the genotype could conceivably play a role in the causal pathway for infant death, its penetrance would have to be very low and probably mediated by other risk factors already identified in the affected populations. 4,5 The second factor related to biological plausibility is that the genotype is associated with reduced, rather than absent, enzyme activity, unlike the rare CPT1A null allele, found in the Hutterite population, for which an association with metabolic crises has been noted. Koeller et al. $^{1}$ argue that the reduction in activity of the CPT1A P479L enzyme compared to the reference sequence is enough to have a significant physiological effect. However, while baseline CPT1A activity of the P479L variant in fibroblasts was found to be just $22 \%$ of the reference sequence, the variant was also associated with a $68 \%$ reduction in the inhibition by malonyl co-A, resulting in residual CPT1A activity that was three to four times that seen in fibroblasts with other CPT1A variants. ${ }^{6}$ Despite these biochemical data, as Koeller et al. note, there is some evidence that the genotype may have a physiological effect. They have observed symptomatic hypoglycemia in two children with the
P479L/P479L genotype subjected to fasting conditions, in an uncontrolled study of five children with the risk genotype. ${ }^{7}$ However, both patients who experienced symptomatic hypoglycemia had a prior history of related symptomsincluding one who had had a seizure with hypoglycemia-and thus they are not representative of children with this genotype, for whom ascertainment has typically been based on tandem mass spectrometry or genotyping, rather than on any clinical presentation. Nevertheless, the observation of symptoms and fasting hypoglycemia in two children with the genotype calls for further study in population-based samples, with careful attention to any other contributing factors.

The second criterion for establishing a causal association that deserves emphasis is the availability of alternative, noncausal, explanations for an association between a specific exposure, in this case the P479L allele, and disease. The populations in which the P479L allele is found are relatively isolated, with living conditions that include high rates of poverty and increased exposure to respiratory pathogens. Maternal social factors associated with infant death are also more prevalent in these populations, including, in addition to poverty, limited access to prenatal care and higher levels of substance abuse. Furthermore, a Canadian study on the association between P479L/P479L and infant death found that all infants with the genotype who died also had risk factors related to sleep practices. ${ }^{8}$ It is thus plausible that the genotype is prevalent on the basis of drift, positive selection, or a founder effect and has a noncausal association with infant death owing to a range of social and environmental risk factors that also occur at higher rates in the population.

We welcome the debate on the level of evidence that is sufficient to warrant public health action. To take public health action, we believe one must have at least strong reason, if not proof, to suppose that the actions to be taken will be beneficial and not cause undue harms. In the case of the statistically significant association between P479L homozygosity and infant death, the proof for direct benefit from monitoring and dietary intervention in infants who are homozygous for the P479L allele is lacking. Without this proof, one must consider all potential causal factors that might contribute to infant death and all associated interventions that might reduce risk. In addition, as we noted in our review, frequent infant feeding may not be helpful in populations currently experiencing increasing rates of obesity and diabetes and could be a source of anxiety or stigma. Certainly more research is needed to determine whether P479L homozygosity plays any part in the causal pathway for infant death and, if so, whether dietary measures can reduce risk. However, the evidence suggests that addressing other risk factors, such as sleeping practices, respiratory pathogen exposure, maternal access to prenatal and postnatal care, poverty, substance abuse, and others that Koeller et al. ${ }^{1}$ 
identify, may have a greater impact on reducing infant mortality than would dietary measures targeting infants homozygous for the P479L allele. Placing the primary focus on dietary interventions could be a serious misdirection of effort and attention.

The educational video released with the support and collaboration of many stakeholder organizations in Alaska reflects the prominence of the P479L allele in public health discourse, and highlights the large impact of any policy that is implemented related to it. While we agree that empowering parents with health information about their children can be an effective route for improving health outcomes, we also believe that policies for doing so must be rooted in strong scientific evidence and in full consideration of their impacts.

The initiation of the comprehensive and collaborative work that Koeller et al. ${ }^{1}$ mention is commended, as more research is undoubtedly needed into the effects of P479L homozygosity, as well as into developing a plan to reduce infant mortality in communities where the variant is commonly found. The recent implementation of DNA testing for the P479L variant, as part of the State of Alaska newborn screening panel, may provide new data to fuel insights into the impacts of P479L homozygosity and potential public health interventions. It should not at this time, however, be characterized as the solution to the pressing public health concern about infant death in Alaska.

\section{DISCLOSURE}

The authors declare no conflict of interest.
Alison E. Fohner, $P h D^{1}$, Nanibaa' A. Garrison, $P h D^{2,3}$, Melissa A. Austin, $\mathrm{PhD}^{4}$ and Wylie Burke, $\mathrm{MD}, \mathrm{PhD}^{5}$

${ }^{1}$ Institute of Public Health Genetics, University of Washington, Seattle, Washington, USA; ${ }^{2}$ Treuman Katz Center for Pediatric Bioethics, Seattle Children's Research Institute, Seattle, Washington, USA; ${ }^{3}$ Department of Pediatrics, Division of Bioethics, School of Medicine, University of Washington, Seattle, Washington, USA; ${ }^{4}$ Department of Epidemiology, University of Washington, Seattle, Washington, USA; ${ }^{5}$ Department of Bioethics and Humanities, School of Medicine, University of Washington, Seattle,

Washington, USA. Correspondence: Alison E. Fohner (afohner@uw.edu)

\section{REFERENCES}

1. Koeller DM, Hirschfeld M, Birch S, et al. Health effects of the CPT1A P479L variant: responsible public health policy. Genet Med 2017;19: e-pub ahead of print 3 August, 2017.

2. Fohner $A E$, Garrison NA, Austin MA, Burke W. Carnitine palmitoyltransferase 1A P479L and infant death: policy implications of emerging data. Genet Med; e-pub ahead of print 26 January 2017.

3. Koepsell TD, Weiss NS. Epidemiologic Methods: Studying the Occurrence of Illness. . Oxford University Press: New York, 2003.

4. Gessner BD, Gillingham MB, Johnson MA, et al. Prevalence and

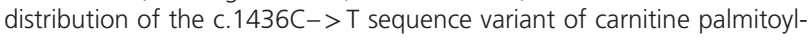
transferase 1A among Alaska Native infants. J Pediatr 2011;158: 124-129.

5. Gessner BD, Gillingham MB, Wood T, Koeller DM. Association of a genetic variant of carnitine palmitoyltransferase $1 \mathrm{~A}$ with infections in Alaska Native children. J Pediatr 2013;163:1716-1721.

6. Brown NF, Mullur RS, Subramanian I, et al. Molecular characterization of L-CPT I deficiency in six patients: insights into function of the native enzyme. J Lipid Res 2001;42:1134-1142.

7. Gillingham MB, Hirschfeld $M$, Lowe $S$, et al. Impaired fasting tolerance among Alaska native children with a common carnitine palmitoyltransferase 1A sequence variant. Mol Genet Metab 2011;104: 261-264.

8. Collins SA, Surmala P, Osborne G, et al. Causes and risk factors for infant mortality in Nunavut, Canada 1999-2011. BMC Pediatr 2012;12:190.

Advance online publication 10 August 2017. doi:10.1038/gim.2017.117 